\title{
Mild cognitive impairment in maintenance hemodialysis patients: a cross-sectional survey and cohort study
}

\author{
Xiaohua Pei ${ }^{1, *}$ \\ Shuyuan Lai ${ }^{1, *}$ \\ Xianglan $\mathrm{He}^{\prime}$ \\ Nakimera Pearl Masembe' \\ Haichuan Yuan' \\ Zhenzhu Yong' \\ Bei Zhu' \\ Jianqing $W^{2}$ \\ Weihong Zhao' \\ 'Department of Geriatric Nephrology, \\ The First Affiliated Hospital of \\ Nanjing Medical University, Nanjing, \\ Jiangsu, China; ${ }^{2}$ Department of \\ Geriatric Respiration, The First \\ Affiliated Hospital of Nanjing Medical \\ University, Nanjing, Jiangsu, China \\ *These authors contributed equally \\ to this work
}

This article was published in the following Dove Press journal: Clinical Interventions in Aging
Correspondence: Weihong Zhao Department of Geriatric Nephrology, The First Affiliated Hospital of Nanjing Medical University, 300 Guangzhou Road, Nanjing 21 0029, Jiangsu, China

Tel +862568305063

Email zhaoweihongny@njmu.edu.cn
Introduction: Few studies focused on mild cognitive impairment (MCI) in maintenance hemodialysis (MHD) patients. This study was conducted to survey the prevalence, the potent risk factors of MCI in MHD patients, and further observe the progress of MCI in a period of 6 months.

Methodology: Mini-Mental State Examination, and Montreal Cognitive Assessment were used to assess cognitive condition. MHD patients were enrolled from The First Affiliated Hospital of Nanjing Medical University, who had a stable hemodialysis history for more than 3 months.

Results: Sixty-four MHD patients and 54 general subjects were finally included. The average age of both groups was more than 60 years. The prevalence of MCI in the MHD group was significantly higher than that in general population $(60.9 \%$ vs $29.6 \%, P<0.05)$. Spearman correlation analysis indicated that $\mathrm{MCI}$ was related to age, comorbidities, education years, uric acid, serum albumin, and blood pressure. The prevalence and severity of MCI in the MHD group remained unchanged during the 6 months (prevalence: $59.5 \%-66.6 \%$, MoCA scores: 22.9-22.5).

Conclusion: MHD patients sustain a fairly high prevalence of MCI. Multiple risk factors influence the incidence and progression of MCI in MHD patients. More attention should be paid to this special population.

Keywords: dementia, end stage renal disease, Montreal Cognitive Assessment, Mini-Mental State Examination

\section{Introduction}

Mild cognitive impairment (MCI), an intermediate state from normal aging to dementia, ${ }^{1}$ has gradually aroused global health professionals' interests. ${ }^{2} \mathrm{McDaniel}$ advocated for the first time in 1971 that cognitive dysfunction may correlate with renal failure. ${ }^{3}$ Then, Hagberg further demonstrated in 1974 that end-stage renal disease (ESRD) and hemodialysis (HD) could be associated with cognitive impairment. ${ }^{4}$ Afterward, Thorleif and Murray proposed that chronic kidney disease (CKD) patients might be more vulnerable to MCI. ${ }^{5,6}$ However, studies focusing on maintenance hemodialysis (MHD) with MCI are just few available.

Thus, this study was designed to observe the prevalence, associated risk factors, and progression of MCI in this specific population. 


\section{Methodology}

\section{Participants}

A total of 64 MHD and 54 general patients in the First Affiliated Hospital of Nanjing Medical University between October 2014 and October 2015 were eventually included (Figure 1). All participants signed informed consents. Ethics committee of the First Affiliated Hospital of Nanjing Medical University approved this study. Inclusion criteria are as follows: MHD - stable and regular HD (three times per week and 4 hours each time) that lasted for more than 3 months, general participants - subjects without CKD and acute kidney injury. Exclusion criteria are as follows: serious neurodegenerative disease and dementia.

\section{$\mathrm{MCl}$ diagnostic criteria}

The diagnostic criteria for MCI referenced the 2010 China guidelines $^{7}$ and the 2014 JAMA adovacacy. ${ }^{8}$ First, MCI was diagnosed through clinical dementia rating and chief complaints. Second, basic activities of daily living (ADL) were reserved, based on the Barthel index scale questionnaire. Third, patients should be free of dementia. These abovementioned judgments and diagnosis were made by a senior neurologist.

\section{Covariates and measurements}

The related clinical characteristics and laboratory parameters were determined, including age, gender, education years,

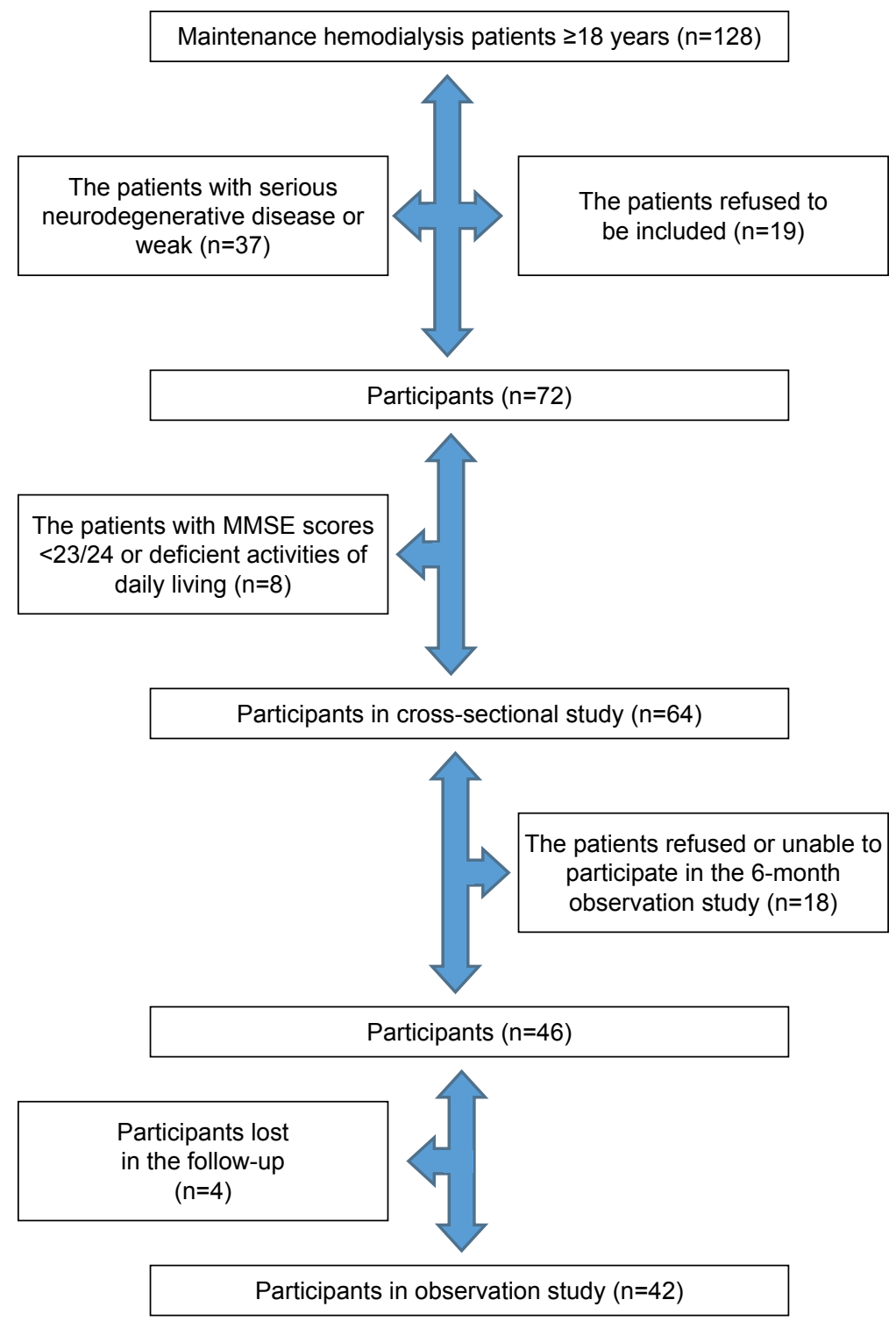

Figure I Flow chart of the subject inclusion.

Abbreviation: MMSE, Mini-Mental State Examination. 
comorbidities, dialysis age, body mass index (BMI), serum creatinine, serum urea nitrogen, uric acid, parathyroid hormone (PTH), albumin, hemoglobin, ferritin, urea reduction ratio (URR), and blood pressure.

\section{Survey and cohort study}

Questionnaires were carried out by the end of each HD treatment. The same questionnaires were repeated again 6 months later.

\section{Statistical analyses}

All the continuous variables were presented as mean \pm SD. Spearman correlation test was applied to ascertain the risk factors of MCI. The McNemar test and Wilcoxon test were measured to understand the progress of MCI. ${ }^{*} P<0.05$ and $* * P<0.01$ indicated statistical significance. All analyses were conducted using SPSS version 20 (IBM Corporation, Armonk, NY, USA).

\section{Results}

\section{Basic characteristics}

Following the inclusion and exclusion criteria, $64 \mathrm{MHD}$ and 54 general patients were finally included. The mean age of both groups was more than 60 years. The dialysis age in the

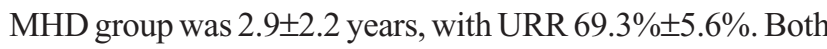
the groups were matched for basic parameters such as age, gender, education years, and complications. The prevalence of lacunar infarction and hypertension in the MHD group was higher than that of the general group but did not reach statistical significance. Additionally, due to renal insufficiency, the concentration of most of the laboratory indexes in the MHD group was much higher than that of the general group, except serum calcium and albumin (Table 1).

\section{Prevalence of $\mathrm{MCl}$ in MHD patients}

The prevalence of MCI in the MHD group was much higher than that in the general group ( $60.9 \%$ vs $29.6 \%$, on the basis of Montreal Cognitive Assessment [MoCA]) with OR 3.71. Mini-Mental State Examination questionnaire indicated that the impaired cognitive functions were the attention and recall parts. MoCA scale showed that MHD patients experienced more serious cognitive deficits, including visuospatial executive function, attention, language, abstraction, and delayed memory (Figure 2).

\section{$\mathrm{MCl}$ risk factors}

Spearman correlation test indicated that age, comorbidities, education years, uric acid, serum albumin, and blood pressure
Table I Basic characteristics of the subjects

\begin{tabular}{|c|c|c|}
\hline Characteristics & $\begin{array}{l}\text { MHD } \\
\text { group } \\
(\mathrm{N}=64)\end{array}$ & $\begin{array}{l}\text { General } \\
\text { participants } \\
(\mathbf{N}=54)\end{array}$ \\
\hline Age (years) & $6 I(17)$ & $65(18)$ \\
\hline Gender (male) & 44 (68.8\%) & 35 (64.8\%) \\
\hline Education (years) & II (4) & $12(4)$ \\
\hline \multicolumn{3}{|l|}{ Comorbidities } \\
\hline Hypertension & 45 (70.3\%) & 32 (59.3\%) \\
\hline Diabetes & $16(25.0 \%)$ & $13(24.1 \%)$ \\
\hline Lacunar infarction & $27(42.2 \%)$ & 21 (38.9\%) \\
\hline \multicolumn{3}{|l|}{ Lab parameters } \\
\hline $\operatorname{Scr}(\mu \mathrm{mol} / \mathrm{L})$ & $863.33(254.61)$ & $73.3(19.57)^{* * *}$ \\
\hline BUN (mmol/L) & $26.6(6.3)$ & $5.5(2.4)^{* * *}$ \\
\hline Uric acid $(\mu \mathrm{mol} / \mathrm{L})$ & $397.2(77.0)$ & $308.8(116.9)^{* * *}$ \\
\hline Potassium (mmol/L) & $5.0(0.7)$ & $4.0(0.4)^{* * *}$ \\
\hline Calcium (mmol/L) & $2.27(0.17)$ & $2.25(0.12)$ \\
\hline Phosphonium (mmol/L) & $1.75(0.54)$ & $\mathrm{I} .06(0.2 \mathrm{I})^{* * * *}$ \\
\hline Albumin (g/L) & $36.5(4.8)$ & $37.5(5.5)$ \\
\hline Hemoglobin $(\mathrm{g} / \mathrm{L})$ & $96.2(14.9)$ & $123.4(20.6)^{* * *}$ \\
\hline BMI $\left(\mathrm{kg} / \mathrm{m}^{2}\right)$ & $21.7(4.2)$ & $23.9(4.0)^{* *}$ \\
\hline $\mathrm{SBP}(\mathrm{mmHg})$ & $144(20)$ & $128(13)^{* * *}$ \\
\hline $\mathrm{DBP}(\mathrm{mmHg})$ & $81(15)$ & $71(12)^{* *}$ \\
\hline PTH (ng/mL) & $328.1(293.3)$ & NR \\
\hline Ferroprotein $(g / L)$ & $290.2(372.3)$ & NR \\
\hline Dialysis ages & $2.9(2.2)$ & NR \\
\hline Urea reduction ratio & $69.3 \%(5.6 \%)$ & NR \\
\hline
\end{tabular}

Notes: Cell values are represented as mean (SD) and $\mathrm{n}(\%) . * * \mathrm{P}<0.01$ and $* * * P<0.001$, compared with the MHD group.

Abbreviations: MHD, maintenance hemodialysis; Scr, serum creatinine; BUN, blood urea nitrogen; BMI, body mass index; PTH, parathyroid hormone; SBP, systolic blood pressure; DBP, diastolic blood pressure; NR, not reported.

were related to MCI in MHD patients, except gender, hemoglobin, ferritin, BMI, PTH, and dialysis age (Table 2).

\section{Development of $\mathrm{MCl}$ with MHD during 6 months}

Forty-two MHD patients consented in a further cohort observation study. The dialysis pattern and volume remained the same. During 6 months, the prevalence and severity of MCI have almost unchanged (prevalence: $59.5 \%-66.6 \%$, MoCA scores: 22.9-22.5) (Table 3 and Figure 3).

\section{Discussion}

MCI, a syndrome with ADL retained and cognitive domain recession, captures increasing attractions. ${ }^{9-11}$ Immense amounts of studies have proved that MCI is the initial stage of dementia. Early diagnosis and intervention of MCI have been recognized as an essential step to prevent the onset of dementia. $^{12}$

Among the previous studies, several researchers proposed that CKD patients are susceptible population of MCI. Along with the decline of renal function, cognitive functions are getting worse. ${ }^{13}$ Dialysis means kidney function paces 


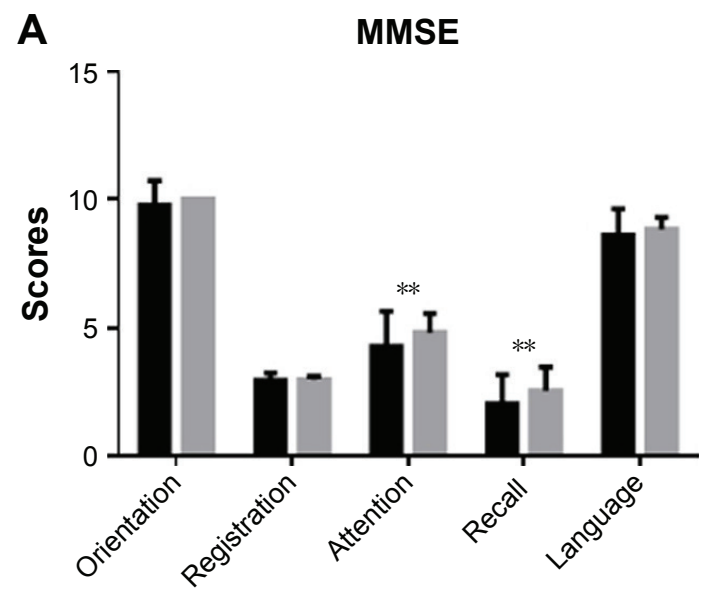

Cognitive domain

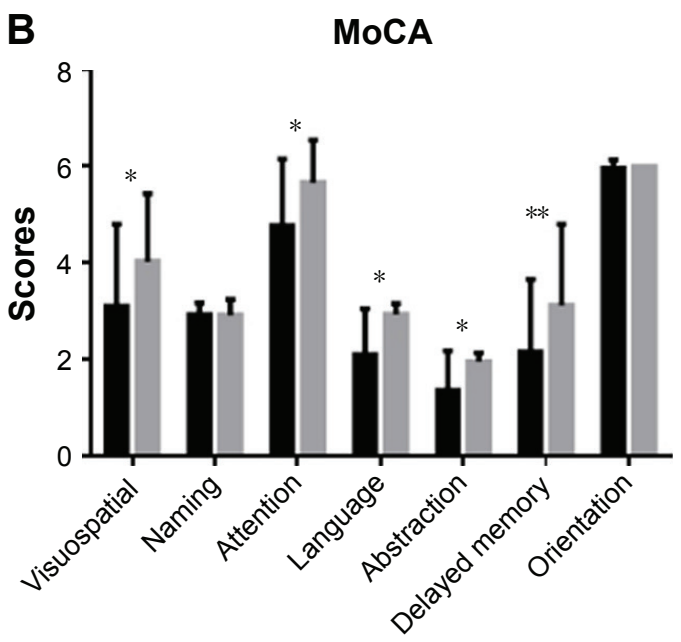

Cognitive domain

MHD group General population

Figure 2 Detailed manifestation of $\mathrm{MCl}$ between the MHD group and general population.

Notes: $(\mathbf{A}$ and $\mathbf{B})$ Vertical coordinates were cognitive domain scores. Horizontal axes were name of cognitive domains. $* P<0.05$ and $* * P<0.01$ indicated statistical significance.

Abbreviations: $\mathrm{MCl}$, mild cognitive impairment; MHD, maintenance hemodialysis; MMSE, Mini-Mental State Examination; MoCA, Montreal Cognitive Assessment.

to the end. For MHD patient, sound cognitive function is of great importance,,$^{14}$ as they have to draw more attention than general patients, to understand, remember, and follow the complicated medical treatments, such as diet control, fluid intake ration, medication therapy, and so on. Once the self-management ability of MHD patients was deprived or impaired, the incidence of tumble and mortality would rapidly increase.${ }^{15}$ However, little if any studies have attached importance to $\mathrm{MCI}$ in the MHD population by far, ${ }^{16}$ especially in China, which will soon be the biggest dialysis country. What are the morbidity rate and the risk factors of MCI in

Table 2 A list of risk factors with $\mathrm{MCl}$

\begin{tabular}{l|l}
\hline Variables & $\boldsymbol{R}$ \\
\hline Age (years) & $-0.597 * * *$ \\
Gender & -0.162 \\
Education years & $0.436 * * *$ \\
Comorbidities & $-0.456 * *$ \\
Uric acid & $0.293^{*}$ \\
Albumin & $0.310^{*}$ \\
PTH & 0.182 \\
Hemoglobin & 0.113 \\
Ferroprotein & 0.046 \\
BMI & -0.184 \\
SBP & $0.280 *$ \\
DBP & $0.293 *$ \\
Dialysis ages & -0.075 \\
\hline
\end{tabular}

Notes: $* P<0.05, * * * P<0.001$. $R$, relationship coefficient.

Abbreviations: $\mathrm{MCl}$, mild cognitive impairment; PTH, parathyroid hormone; BMI, body mass index; SBP, systolic blood pressure; DBP, diastolic blood pressure.
MHD patients? How to effectively delay the MCI process for dialysis patients?

Our results showed that the prevalence of MCI in MHD patients $(60.9 \%)$ was twice as many as general population (29.5\%), which was similar to the previous reports. ${ }^{17}$ Therefore, a fairly high prevalence of $\mathrm{MCI}$ is indeed ubiquitous in the MHD patients. So, what are the reasons that bring about such a high prevalence? Our results revealed that apart from the traditional causes, such as aging and education years, comorbidities, uric acid, serum albumin, and blood pressure were also related to the occurrence of MCI in MHD patients. As shown in our results, previous studies also found that for dialysis population, other than common risk factors, uremic toxin accumulation, ${ }^{18}$ vascular endothelial injury, hormone deficiency, malnutrition, blood exposure, anemia, and dyslipidemia were closely related to cognition dysfunction. ${ }^{19}$ Uric acid and blood pressure were independent risk factors with MCI. ${ }^{20}$ Thereout, MHD patients have more exposures to susceptibility factors of MCI. To efficiently prevent the

Table 3 Prevalence and severity of $\mathrm{MCl}$ in $\mathrm{MHD}$ patients after 6 months

\begin{tabular}{l|l|l}
\hline & Baseline & 6 months later \\
\hline $\mathrm{MCl}$ prevalence & $25(59.5 \%)$ & $28(66.6 \%)$ \\
MoCA scores & $22.9(5.4)$ & $22.5(5.9)$ \\
\hline
\end{tabular}

Notes: Cell values represent mean (SD) and $n(\%)$. The cutoff value of MoCA was 26.

Abbreviations: $\mathrm{MCl}$, mild cognitive impairment; $\mathrm{MHD}$, maintenance hemodialysis; MoCA, Montreal Cognitive Assessment. 
A

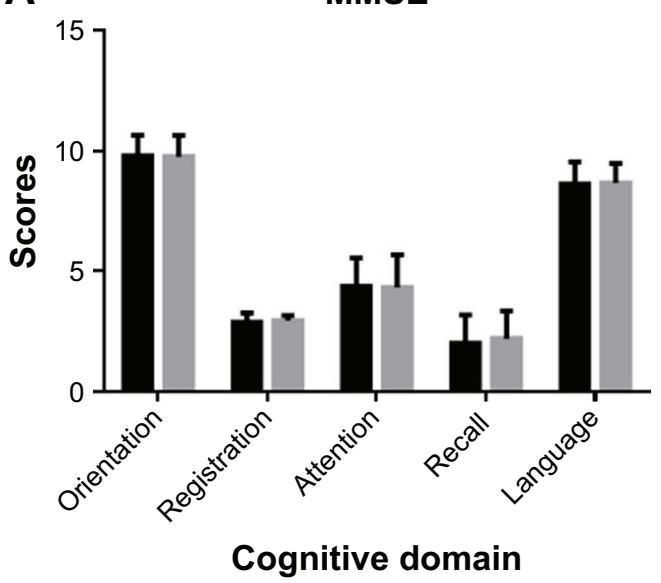

B

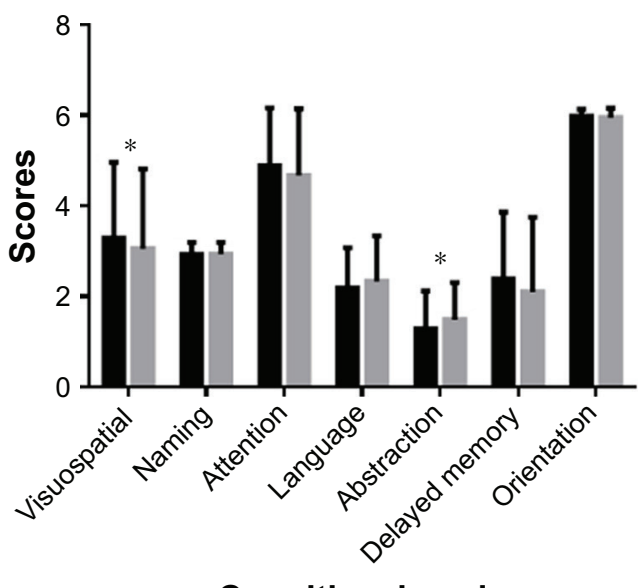

Baseline

6 months later

Figure 3 Progress of $\mathrm{MCl}$ with MHD during 6 months.

Notes: (A and B) Vertical coordinates were cognitive domain scores. Horizontal axes were name of cognitive domains. $* P<0.05$ indicated statistical significance.

Abbreviations: $\mathrm{MCl}$, mild cognitive impairment; MHD, maintenance hemodialysis; MMSE, Mini-Mental State Examination; MoCA, Montreal Cognitive Assessment.

onset of MCI, more attention should be paid and various measures should be taken.

The first and foremost way is to screen cerebrovascular event, which is a familiar and vital reason for cognition dysfunction. $^{21,22}$ The onset of MCI could always be along with the occurrence of lacunar strokes. The phosphodiesterase III inhibitor cilostazol, which is often used in the prevention of stroke ${ }^{23}$ and peripheral artery disease, ${ }^{24}$ may delay cognitive decline. ${ }^{25}$ There is one more method that should be touched on; once they are diagnosed as MCI, cognitive enhancers, including cholinesterase inhibitors ${ }^{26}$ and memantine, ${ }^{27}$ are recommended to treat the disease. Last but not the least, crucial measure should focus on the dialysis duration, dialysis adequacy, and dialysis pattern. A longer HD duration may correlate with a lower dementia risk, based on an analysis of over 200 thousand HD patients in Japan. ${ }^{28}$ Frequent HD schedules might associate with reduced levels of dialysis-induced cardiac injury. ${ }^{29}$ Dialysis adequacy is calculated based on small molecular uremic toxin (urea). But comorbidities such as cardiovascular disease and neuropathy are caused mainly by middle molecular and protein-bound uremic toxin. So, the current dialysis techniques cannot improve patient prognosis further, ${ }^{30}$ which had also been elucidated in the HEMO study. Daily HD or nocturnal dialysis may have no benefit, compared with ordinal HD. ${ }^{31}$

\section{Conclusion}

MHD patients are prone to MCI, and great concerns should be focused on this specific population. Susceptible factors in
MHD patients were more complicated than those in general population. A longer prospective cohort study with more subjects might provide more credible information. ${ }^{32}$

\section{Acknowledgments}

We thank all the staff of the Department of Geriatric Nephrology, The First Affiliated Hospital of Nanjing Medical University, for their cooperation and the anonymous reviewers. This work was supported by the grants from Clinical Medicine Research Special Funds of Chinese Medical Association 15020020590, Jiangsu Provincial Key Discipline of Medicine ZDXKA2016003, Jiangsu Province's Key Medical Talents Program ZDRCA2016021, Jiangsu Province 333 Project BRA2017409, Jiangsu Province's Key Medical Young Talents Program QNRC2016592, and Jiangsu Cadres Health Care Research BJ16016.

\section{Author contributions}

WHZ conceived and designed the study. JQW guided and advanced the study. SYL, XHP, XLH, and HCY performed the experiments. XHP, SYL, ZZY, and BZ analyzed the experimental data. XHP, SYL, and NPM wrote the manuscript. XHP and WHZ reviewed and edited the manuscript. All authors contributed to data analysis, drafting and revising the article, gave final approval of the version to be published, and agree to be accountable for all aspects of the work.

\section{Disclosure}

The authors report no conflicts of interest in this work. 


\section{References}

1. Mcguinness B, Barrett SL, Mcilvenna J, Passmore AP, Shorter GW. Predicting conversion to dementia in a memory clinic: a standard clinical approach compared with an empirically defined clustering method (latent profile analysis) for mild cognitive impairment subtyping. Alzheimers Dement. 2015;1(4):447-454.

2. González-Vélez AE, Forjaz MJ, Giraldez-García C, Martín-García S, Martínez-Martín P; Spanish Research Group on Quality of Life and Ageing. Quality of life by proxy and mortality in institutionalized older adults with dementia. Geriatr Gerontol Int. 2015;15(1):38-44.

3. Mcdaniel JW. Metabolic and CNS correlates of cognitive dysfunction with renal failure. Psychophysiology. 1971;8(6):704-713.

4. Hagberg B. A prospective study of patients in chronic hemodialysisIII. Predictive value of intelligence, cognitive deficit and ego defence structures in rehabilitation. J Psychosom Res. 1974;18(3):151-160.

5. Thorleif E, Michel C, Hans F, Dirk S. Chronic kidney disease and cognitive impairment: a systematic review and meta-analysis. $\mathrm{Am} \mathrm{J}$ Nephrol. 2012;35:474-482.

6. Murray AM, Tupper DE, Knopman DS, et al. Cognitive impairment in hemodialysis patients is common. Neurology. 2006;67(2):216-223.

7. Writing Goup of the Dementia and Cognitive Society of Neurology Committee of Chinese Medical AssociationAlzheimer's Disease Chinese. Guidelines for dementia and cognitive impairment in China: the diagnosis and treatment of mild cognitive impairment. Zhonghua Yi Xue Za Zhi. 2010;90(41):2887-2893.

8. Langa KM, Levine DA. The diagnosis and management of mild cognitive impairment: a clinical review. JAMA. 2014;312(23):2551-2561.

9. Honig LS, Vellas B, Woodward M, et al. Trial of solanezumab for mild dementia due to Alzheimer's disease. N Engl J Med. 2018;378(4): 321-330.

10. Schmidt LI, Wahl HW. Predictors of performance in everyday technology tasks in older adults with and without mild cognitive impairment. Gerontologist. Epub 2018 Jun 06.

11. Ströhle A, Schmidt DK, Schultz F, et al. Drug and exercise treatment of alzheimer disease and mild cognitive impairment: a systematic review and meta-analysis of effects on cognition in randomized controlled trials. Am J Geriatr Psychiatry. 2015;23(12):1234-1249.

12. Colangeli S, Boccia M, Verde P, Guariglia P, Bianchini F, Piccardi L. Cognitive reserve in healthy aging and Alzheimer's disease: a meta-analysis of FMRI studies. Am J Alzheimers Dis Other Demen. 2016; 31(5):443-449.

13. Yaffe K, Ackerson L, Kurella Tamura M, et al. Chronic Renal Insufficiency Cohort Investigators. Chronic kidney disease and cognitive function in older adults: findings from the chronic renal insufficiency cohort cognitive study. J Am Geriatr Soc. 2010;58(2):338-345.

14. O'Lone E, Connors M, Masson P, et al. Cognition in people with endstage kidney disease treated with hemodialysis: a systematic review and meta-analysis. Am J Kidney Dis. 2016;67(6):925-935.

15. Han SS, Park JY, Kang S, et al. Dialysis modality and mortality in the elderly: a meta-analysis. Clin J Am Soc Nephrol. 2015;10(6): 983-993.

16. Nie $\mathrm{H}, \mathrm{Xu} \mathrm{Y}$, Liu B, et al. The prevalence of mild cognitive impairment about elderly population in China: a meta-analysis. Int J Geriatr Psychiatry. 2011;26(6):558-563.
17. Ma F, Wu T, Zhao J, et al. Prevalence of mild cognitive impairment and its subtypes among Chinese older adults: role of vascular risk factors. Dement Geriatr Cogn Disord. 2016;41(5-6):261-272.

18. Watanabe K, Watanabe T, Nakayama M. Cerebro-renal interactions: impact of uremic toxins on cognitive function. Neurotoxicology. 2014;44:184-193.

19. Eldehni MT, Odudu A, Mcintyre CW. Randomized clinical trial of dialysate cooling and effects on brain white matter. J Am Soc Nephrol. 2015;26(4):957-965.

20. Afsar B, Elsurer R, Covic A, Johnson RJ, Kanbay M. Relationship between uric acid and subtle cognitive dysfunction in chronic kidney disease. Am J Nephrol. 2011;34(1):49-54.

21. Lim EY, Yang DW, Cho AH, Shim YS. Cerebrovascular hemodynamics on transcranial doppler ultrasonography and cognitive decline in mild cognitive impairment. J Alzheimers Dis. 2018;65(2):651-657.

22. Makin SD, Turpin S, Dennis MS, Wardlaw JM. Cognitive impairment after lacunar stroke: systematic review and meta-analysis of incidence, prevalence and comparison with other stroke subtypes. J Neurol Neurosurg Psychiatry. 2013;84(8):893-900.

23. Toyoda K, Uchiyama S, Hoshino H, et al. Protocol for Cilostazol Stroke Prevention Study for Antiplatelet Combination (CSPS. com): a randomized, open-label, parallel-group trial. Int J Stroke. 2015;10(2):253-258.

24. Soga Y, Hamasaki T, Edahiro R, et al. Sustained effectiveness of cilostazol after endovascular treatment of femoropopliteal lesions: midterm follow-up from the sufficient treatment of peripheral intervention by cilostazol (STOP-IC) Study. J Endovasc Ther. 2018;25(3):306-312.

25. Saito S, Kojima S, Oishi N, et al. A multicenter, randomized, placebo-controlled trial for cilostazol in patients with mild cognitive impairment: The COMCID study protocol. Alzheimers Dement. 2016;2(4):250-257.

26. Colombo D, Caltagirone C, Padovani A, et al. Gender differences in neuropsychiatric symptoms in mild to moderate Alzheimer's disease patients undergoing switch of cholinesterase inhibitors: a post hoc analysis of the EVOLUTION study. J Womens Health. Epub 2018 Sep 08.

27. Ghaffary S, Ghaeli P, Talasaz AH, et al. Effect of memantine on postoperative cognitive dysfunction after cardiac surgeries: a randomized clinical trial. Daru. 2017;25(1):24.

28. Nakai S, Wakai K, Kanda E, Kawaguchi K, Sakai K, Kitaguchi N. Is hemodialysis itself a risk factor for dementia? An analysis of nationwide registry data of patients on maintenance hemodialysis in Japan. Renal Replacement Therapy. 2018;4:12.

29. Jefferies HJ1, Virk B, Schiller B, Moran J, McIntyre CW. Frequent hemodialysis schedules are associated with reduced levels of dialysisinduced cardiac injury (myocardial stunning). Clin J Am Soc Nephrol. 2011;6(6):1326-1332.

30. Giang LM, Weiner DE, Agganis BT, et al. Cognitive function and dialysis adequacy: no clear relationship. Am J Nephrol. 2011;33(1):33-38.

31. Kurella Tamura M, Unruh ML, Nissenson AR, et al. Effect of more frequent hemodialysis on cognitive function in the frequent hemodialysis network trials. Am J Kidney Dis. 2013;61(2):228-237.

32. Kitaguchi N, Hasegawa M, Ito S, et al. A prospective study on blood $\mathrm{A} \beta$ levels and the cognitive function of patients with hemodialysis: a potential therapeutic strategy for Alzheimer's disease. JNeural Transm. 2015;122(11):1593-1607.
Clinical Interventions in Aging

\section{Publish your work in this journal}

Clinical Interventions in Aging is an international, peer-reviewed journal focusing on evidence-based reports on the value or lack thereof of treatments intended to prevent or delay the onset of maladaptive correlates of aging in human beings. This journal is indexed on PubMed Central, MedLine,

\section{Dovepress}

CAS, Scopus and the Elsevier Bibliographic databases. The manuscript management system is completely online and includes a very quick and fair peer-review system, which is all easy to use. Visit http://www.dovepress. com/testimonials.php to read real quotes from published authors. 